\title{
The perfluorinated trityl cation accessible as a triflate
}

\section{derivative}

Eoghan G. Delany, ${ }^{a}$ Satnam Kaur, ${ }^{a}$ Steven Cummings ${ }^{c}$, Kristoffer Basse, ${ }^{b}$ David J. D. Wilson, ${ }^{a}$ and Jason L. Dutton ${ }^{a *}$

${ }^{a}$ Department of Chemistry and Physics, La Trobe Institute for Molecular Science, La Trobe University, Melbourne, Victoria, Australia, 3086

${ }^{\mathrm{b}}$ La Trobe University Comprehensive Proteomics Platform, Melbourne, Victoria, Australia, 3086

${ }^{c}$ Department of Chemistry, Howard University, 525 College St, NW, Washington, DC 20059.

\begin{abstract}
While ultimately not isolable for X-ray structural characterization, the free perfluorinated trityl cation is shown to be observable in neat triflic acid, which represents milder conditions than previous reports of this cation in "magic acid" or oleum. A triflate-bound species can be generated in organic solvents using stoichiometric amounts of triflic acid and is shown to be synthetically viable for hydride abstraction from $\mathrm{Et}_{3} \mathrm{SiH}$. It is demonstrated that the para position on the $-\mathrm{C}_{6} \mathrm{~F}_{5}$ rings is the primary point of attack for decomposition of the cation.
\end{abstract}




\section{INTRODUCTION}

The perfluorinated analogue of the trityl cation $\left(\mathbf{1}^{+}\right)$was first observed in superacidic media ${ }^{1}$ independently by Filler ${ }^{2}$ and by $\mathrm{Olah}^{3}$ in the 1960 s, generated by protonation of an alcohol precursor (10H, Scheme 1). The stability of $\mathbf{1}^{+}$was limited in oleum, better in "magic acid", but no salt of the cation could be isolated.
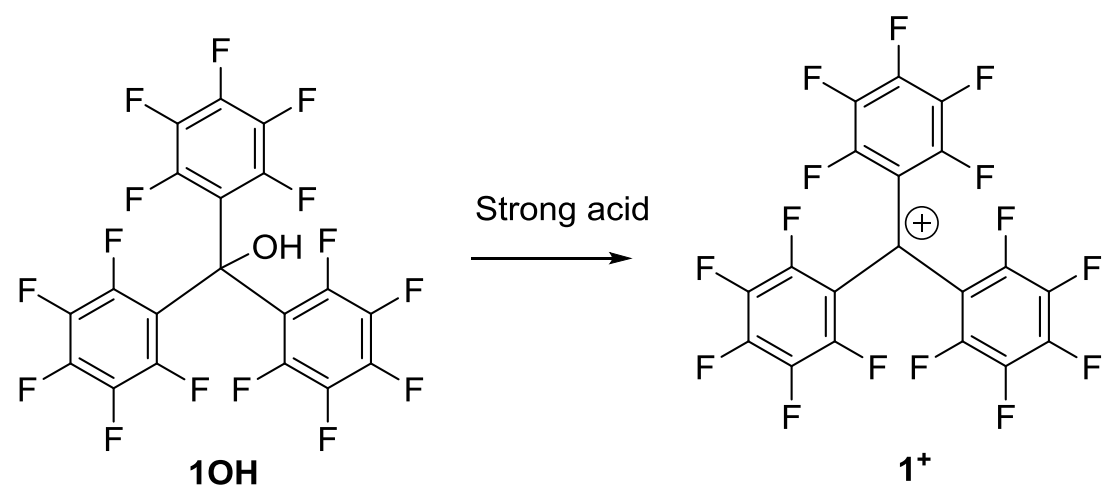

Scheme 1. Generation of trityl cation $\mathbf{1}^{+}$under superacidic conditions.

The electrophilicities of partially fluorinated trityl cations with have been studied recently by Mayr and co-workers, ${ }^{4,5}$ but the fully fluorinated analogue was not included. Given the explosion in utility observed for the boron counterpart, trispentafluorophenyl borane, ${ }^{6,7}$ and the availability of new counterions for reactive cations ${ }^{8,9}$ we proposed in a theoretical paper in 2014 that it was time to revisit the fully fluorinated analogue of the trityl cation. ${ }^{10}$ We predicted that its fluoride and hydride affinities would be very high, much higher than those of trityl cation and trispentafluorophenyl borane. Stephan and co-workers studied the perfluorinated trityl cation as an extreme Lewis acid showing good correlation between predicted Global Electrophilicity Indexes and Lewis acidity. ${ }^{11}$

Herein we report on our efforts to observe and isolate cation $\mathbf{1}^{+}$as a triflate or carborane salt in nonsuperacidic media along with an exploration of its chemistry. 


\section{RESULTS AND DISCUSSION}

Our initial goal was to prepare a halo derivative of $\mathbf{1 0 H}$ for use in halide abstraction routes to form $\mathbf{1}^{+}$. The starting alcohol $\mathbf{1 0 H}$ was initially synthesized by Filler et al. by reacting $\mathrm{LiC}_{6} \mathrm{~F}_{5}$ with decafluorobenzophenone. ${ }^{2}$ They reported being unable to synthesize a halogenated derivative. We repeated their synthesis of $\mathbf{1 0 H}$ and further verified its structure by X-ray (Figure 1). Caution: in the course of a routine $2 \mathrm{~g}$ synthesis, we experienced an explosion from $\mathrm{LiC}_{6} \mathrm{~F}_{5}$ despite the mixture being held in a dry-ice/EtOH bath. We subsequently found that replacing the lithium reagent with the Grignard reagent $\mathrm{BrMg}-\mathrm{C}_{6} \mathrm{~F}_{5}$, generated from $\mathrm{Mg}$ metal and $\mathrm{Br}-\mathrm{C}_{6} \mathrm{~F}_{5}$, provides a suitable alternative route to $\mathbf{1 0 H}$, albeit with a somewhat lower yield.

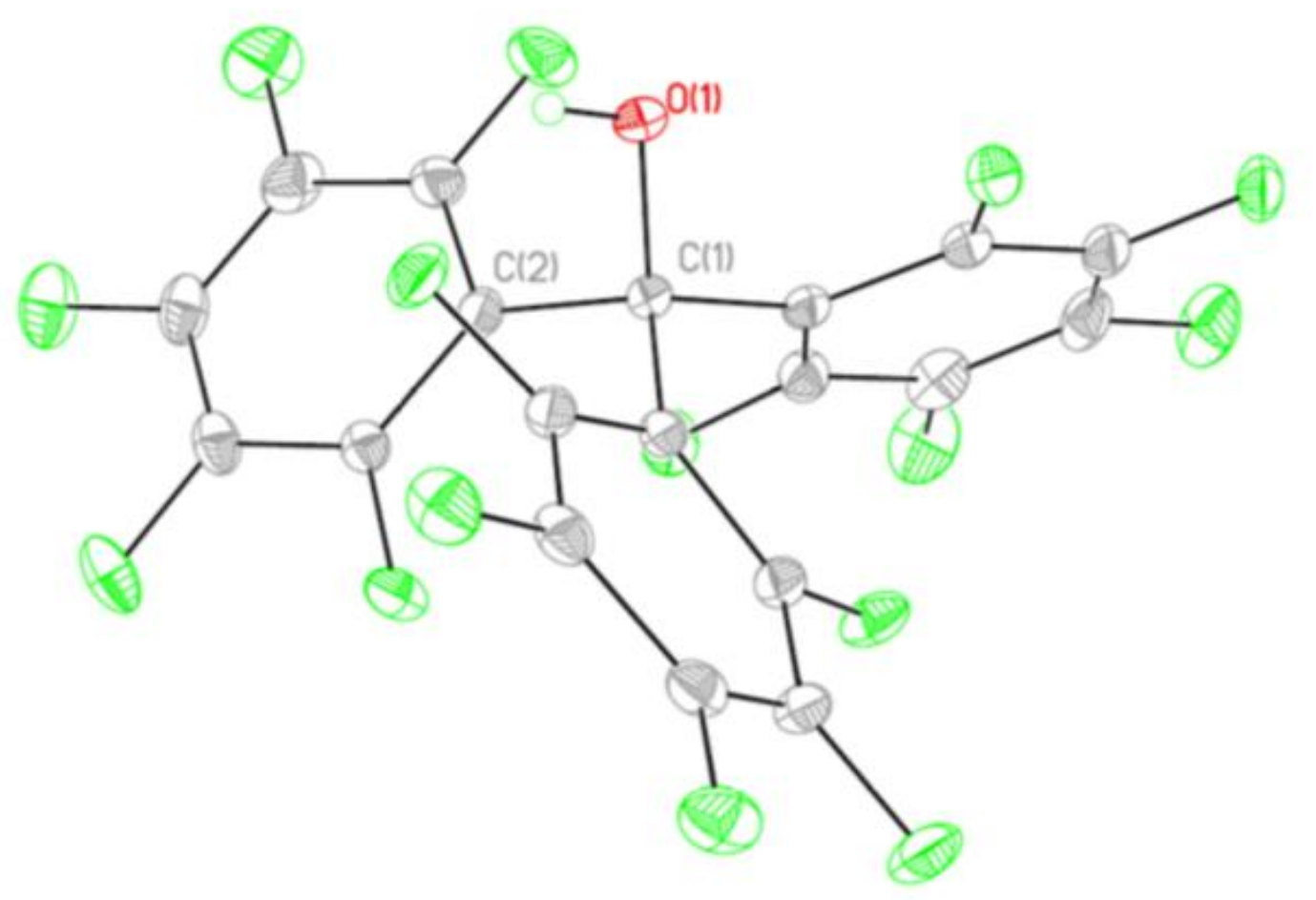


Figure 1. Solid-state structure of $\mathbf{1 0 H}$. Thermal ellipsoids are depicted at the $50 \%$ probability level.

In an attempt to form the bromo derivative, $\mathbf{1 0 H}$ was reacted with neat acetyl bromide, following the reported procedure for the formation of trityl bromide from trityl alcohol. ${ }^{12}$ Fluorine-19 NMR of the reaction mixture and isolated material gave the expected three resonances, shifted from those from 10H. However, X-ray studies revealed the presence of the acetate derivative, 10Ac, rather than the targeted bromide (Figure 2). The ${ }^{1} \mathrm{H}$ NMR spectrum of the isolated powder contained a singlet, confirming identity of the bulk material with the single crystal.

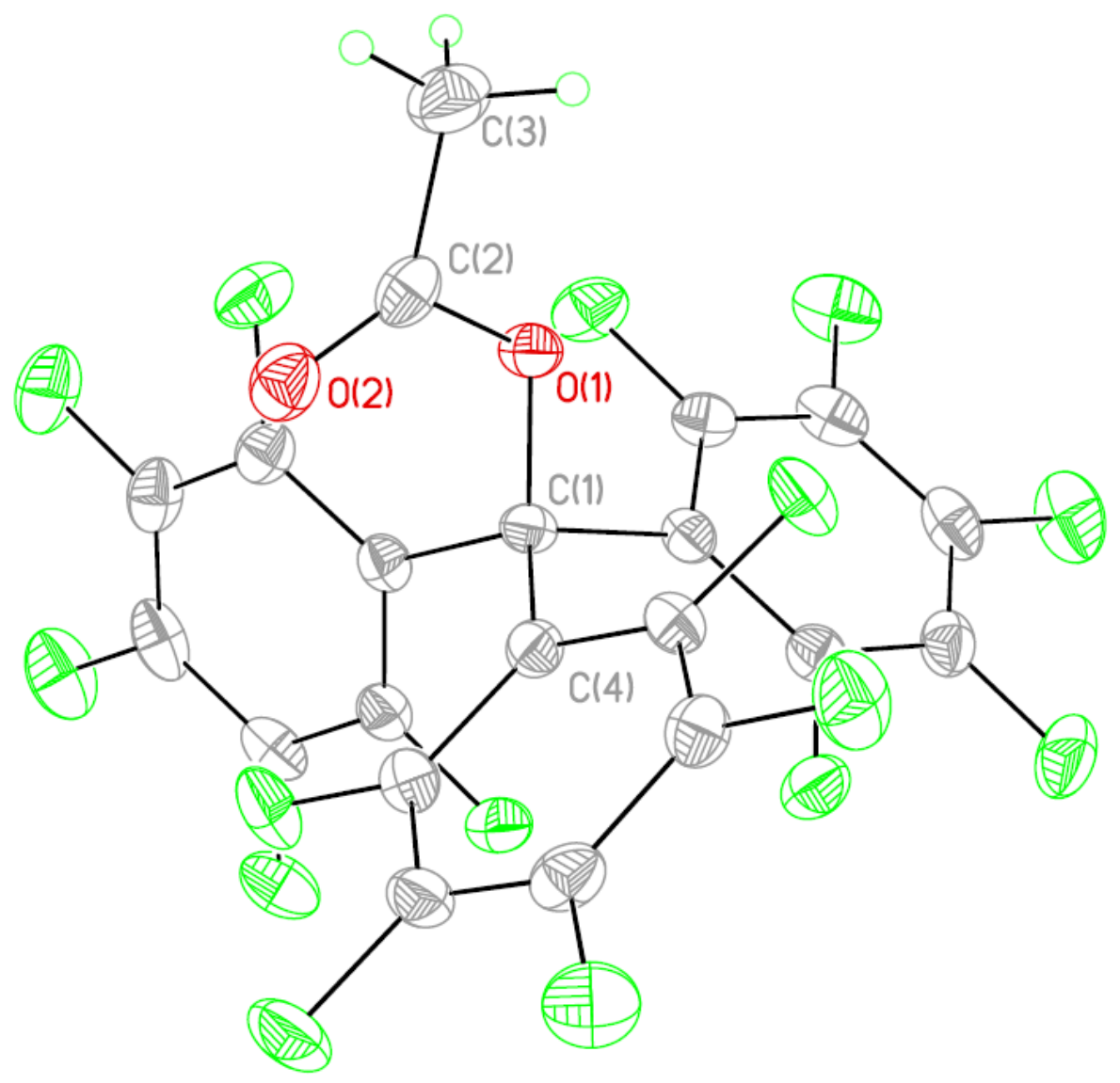

Figure 2. Solid-state structure of 10Ac. Thermal ellipsoids are depicted at the $50 \%$ probability level. 
We next used $\mathrm{SOCl}_{2}$ to form the chloro derivative (Scheme 2). Reaction in $p$-xylene at $142{ }^{\circ} \mathrm{C}$ consistently resulted in a crude product showing nine ${ }^{19} \mathrm{~F}$ NMR signals. The mixture could be chromatographically separated into two compounds, one showing six signals, the other showing the expected three signals from symmetric $-\mathrm{C}_{6} \mathrm{~F}_{5}$ groups. X-ray diffraction revealed the latter structure to be the targeted chloroalkane $\mathbf{1 C l}$ (Figure 3). The former compound, with ${ }^{2}{ }^{19} \mathrm{~F}$ signals, was revealed to be-chlorinated at the para-position with $\mathrm{sp}^{3}$ hybridized carbon, 2 (Figure 4). Heating $\mathbf{1 C l}$ to high temperatures results in transformation to $\mathbf{2}$, presumably by attack from chlorine onto the para position, which is known to be a reactive site on $-\mathrm{C}_{6} \mathrm{~F}_{5}$ rings with respect to nucleophilic attack. ${ }^{13}$ Efforts to minimize formation of this para-substituted isomer through various synthetic modifications (lower reaction temperatures, Vilsmeier-Haack chemistry or alternative chlorinating agents) failed to give better yields of the desired $\mathbf{1 C l}$. Indeed, 2 often became the sole product. This suggests product formation is under kinetic control, favoring less sterically hindered attack of the chloride ion at the para position. Nevertheless, under milder reaction temperatures $\left(\leq 80{ }^{\circ} \mathrm{C}\right.$ approx.) 2 shows significantly reduced solubility relative to the desired chloroalkane, allowing isolation of $\mathbf{1 C l}$ in reasonable purity.
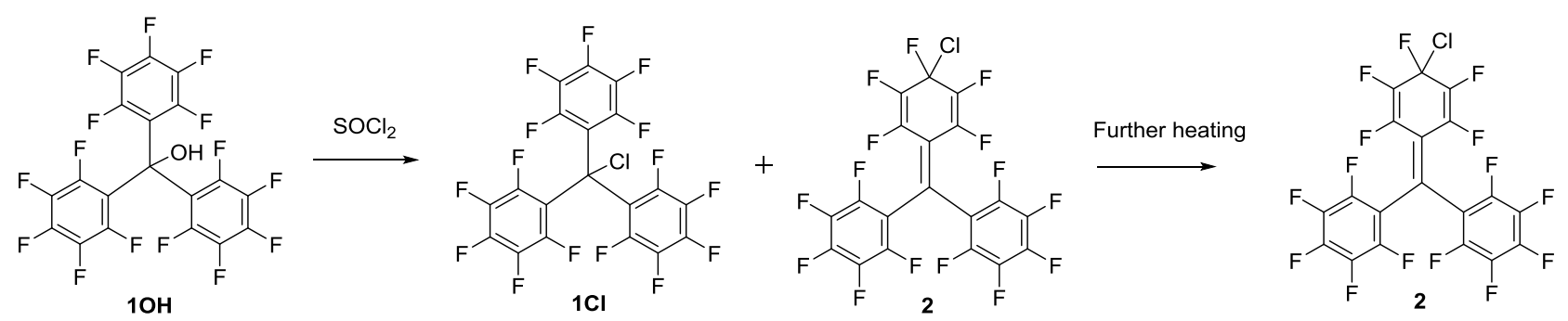

Scheme 2. Generation of chloro-derivatives $\mathbf{1 C l}$ and 2.

Examination of the solid-state contacts in $\mathbf{1 C l}$ shows that the intermolecular contact for the chloride atom and the para carbon position is $3.35 \AA$, less than the sum of their Van der Waals radii of $\sim 3.5$ 
$\AA$. These are the closest contacts for these atoms, indicative of the Lewis basic nature of the terminal chloride and more importantly, the Lewis acidic nature of the para carbon atom.

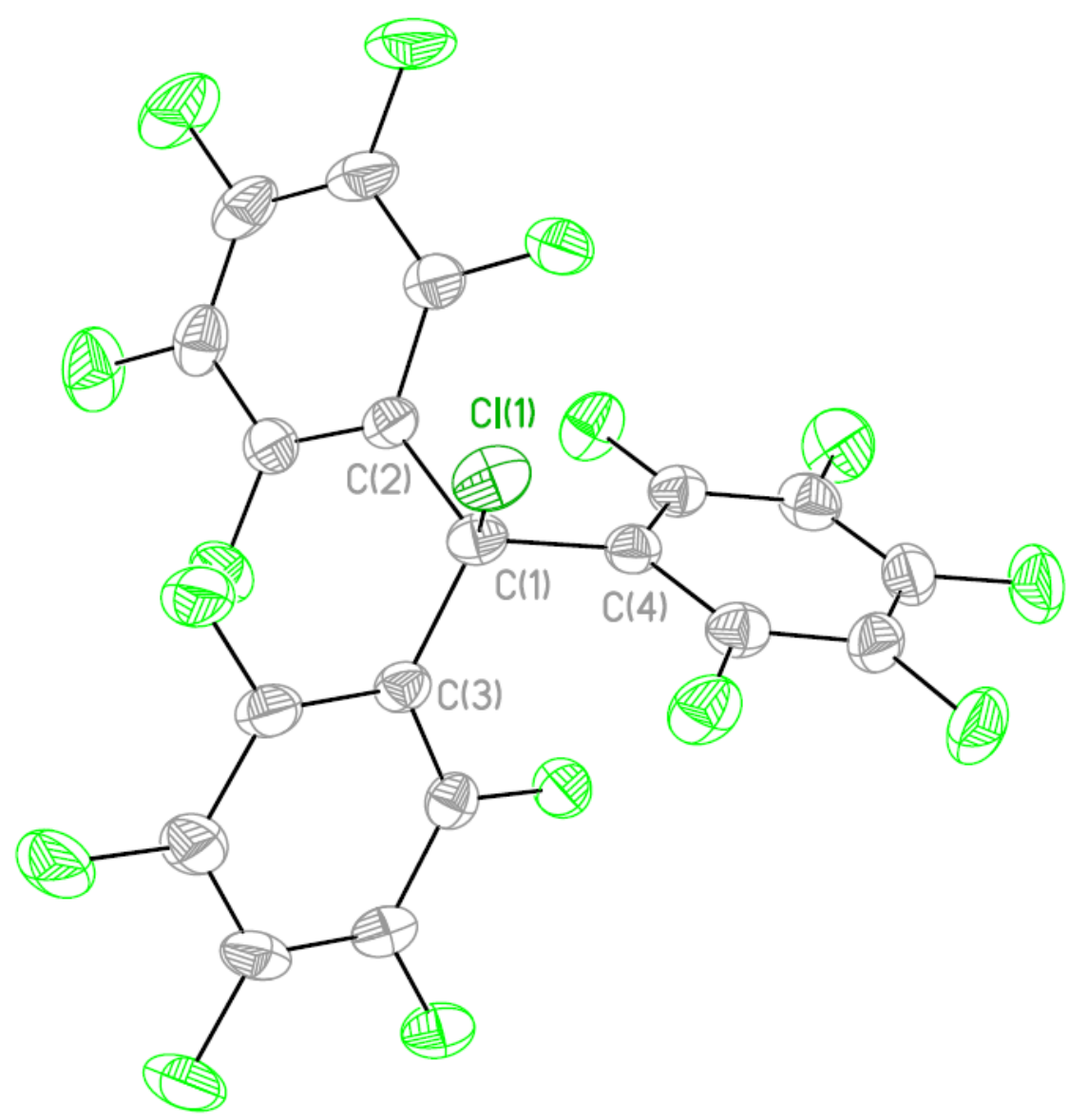

Figure 3. Solid-state structure of $\mathbf{1 C l}$. Thermal ellipsoids are depicted at the $50 \%$ probability level. Selected bond distances ( $\mathrm{A})$ : $\mathrm{C}(1)-\mathrm{Cl}(1)$ 1.845(2), C(1)-C(4) 1.538(3). 


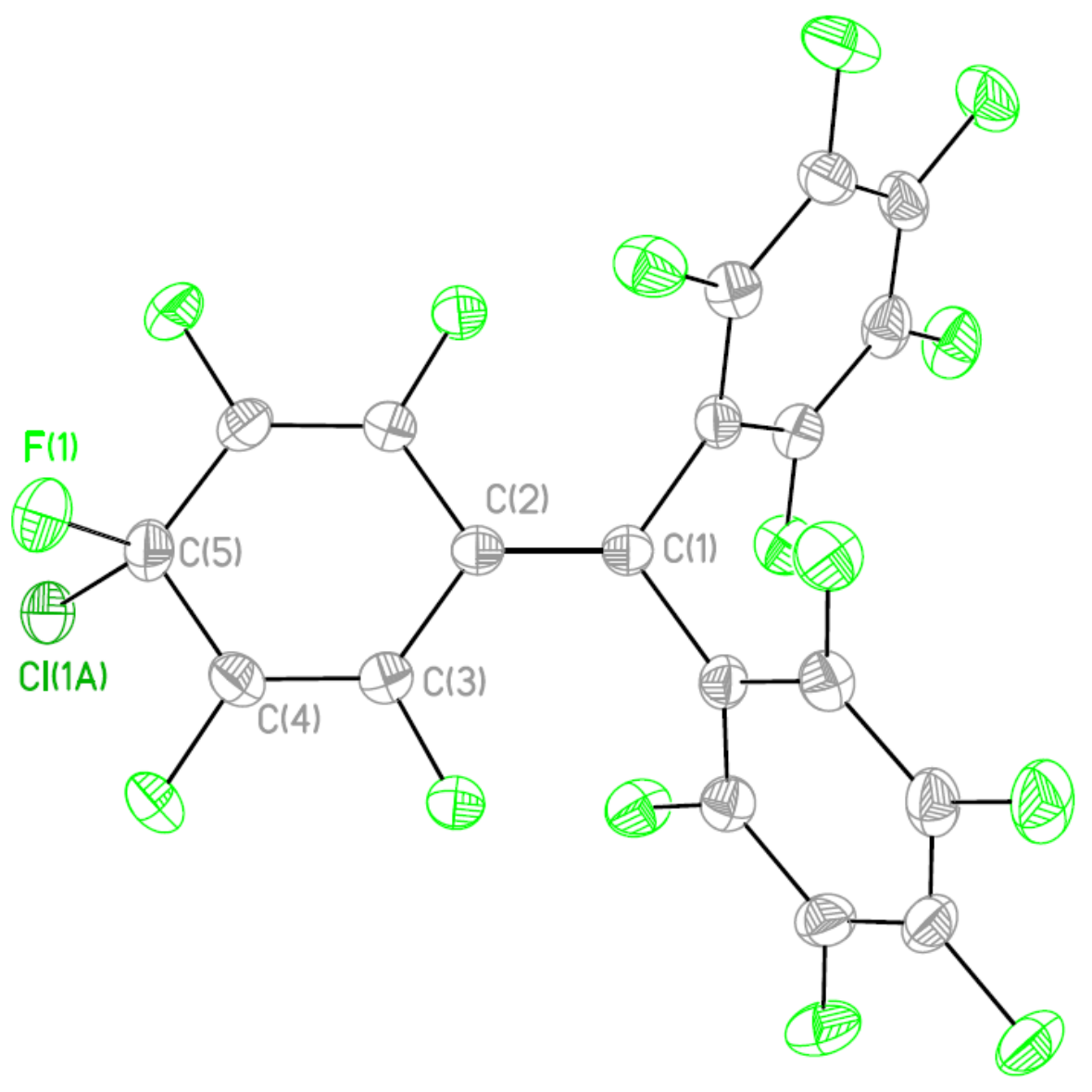

Figure 4. Solid-state structure of 2. Thermal ellipsoids are depicted at the 50\% probability level. Selected bond distances ( $\AA$ ): C(5)-Cl(1A) 1.727(3), C(5)-F(1) 1.415(5), C(4)-C(5) 1.484(2), C(3)$\mathrm{C}(4) 1.327(2), \mathrm{C}(2)-\mathrm{C}(3) 1.466(2), \mathrm{C}(1)-\mathrm{C}(2) 1.355(3)$.

With the requisite chloroalkane in hand, we set about investigating its reaction with various halide abstraction agents. ${ }^{19} \mathrm{~F}$ NMR analysis of a 1:1 mixture of $\mathbf{1 C l}$ and silver triflate (AgOTf) after stirring at ambient temperature for 20 hours in acetonitrile indicated formation of a new species having similar characteristics to the starting material. However, concentration of the colourless reaction mixture resulted in isolation of a new red solid whose ${ }^{19} \mathrm{~F}$ NMR showed five new resonances. X-ray analysis revealed this compound to be the dienone 6 (Scheme 3, Figure 5), previously reported from quenching reactions between $\mathbf{1 O H}$ and $\mathrm{H}_{2} \mathrm{SO}_{4}$ with water. ${ }^{14}$ Although 
employing stringent anhydrous conditions, we surmised that trace amounts of water lead to its formation. Thus, despite all reasonable efforts to exclude water from the system, we continued to isolate $\mathbf{6}$ as the sole reaction product- The same outcome was observed when employing a stoichiometric amount of trimethylsilyl triflate (TMSOTf) instead of AgOTf. Attempts at using $\mathrm{Ag}_{2}\left[\mathrm{~B}_{12} \mathrm{Cl}_{12}\right]$ as a halide abstraction agent introducing the weakly coordinating $\left[\mathrm{B}_{12} \mathrm{Cl}_{12}\right]^{2-}$ anion ${ }^{15}$ resulted in no reaction.

A plausible reaction pathway to rationalize this reproducible formation of $\mathbf{6}$ is proposed in Scheme 3. In solution, chloroalkane $\mathbf{1 C l}$ undergoes gradual conversion to its para-chloro isomer $\mathbf{2}$ (vida supra). Similarly, the kinetically-controlled attack of a nucleophile (in this case, water or triflate anion) at the para position of one perfluorinated aryl ring results in formation of alkene 4 through loss of leaving group X. In this case, the leaving group may be a chloride ion or hydroxyl/triflate group (given the appearance of resonances correlating well to the perfluorinated alcohol in crude ${ }^{19} \mathrm{~F}$ NMR spectra). Subsequent elimination of $\mathrm{F}^{-}$yields the more stable conjugated dienone $\mathbf{6}$ via $\mathbf{5}$.<smiles>[R]OCCCCC([X])(F)c1c(F)c(F)c(F)c(F)c1F</smiles>

3

$\mathrm{R}=\mathrm{H}$ or $\mathrm{SOCF}_{3}$ $\mathrm{X}=\mathrm{Cl}, \mathrm{OH}$ or $\mathrm{OSOCF}_{3}$

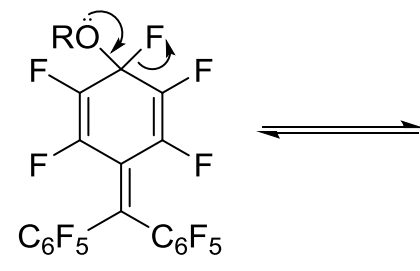

4<smiles>[R][O+]=C1C(F)=C(F)C(=C(C(F)(F)F)C(F)(F)F)C(F)=C1F</smiles>

5<smiles>O=C1C(F)=C(F)C(=C(C(F)(F)F)C(F)(F)F)C(F)=C1F</smiles>

6

Scheme 3. Proposed mechanism for the generation of ketone 6. 


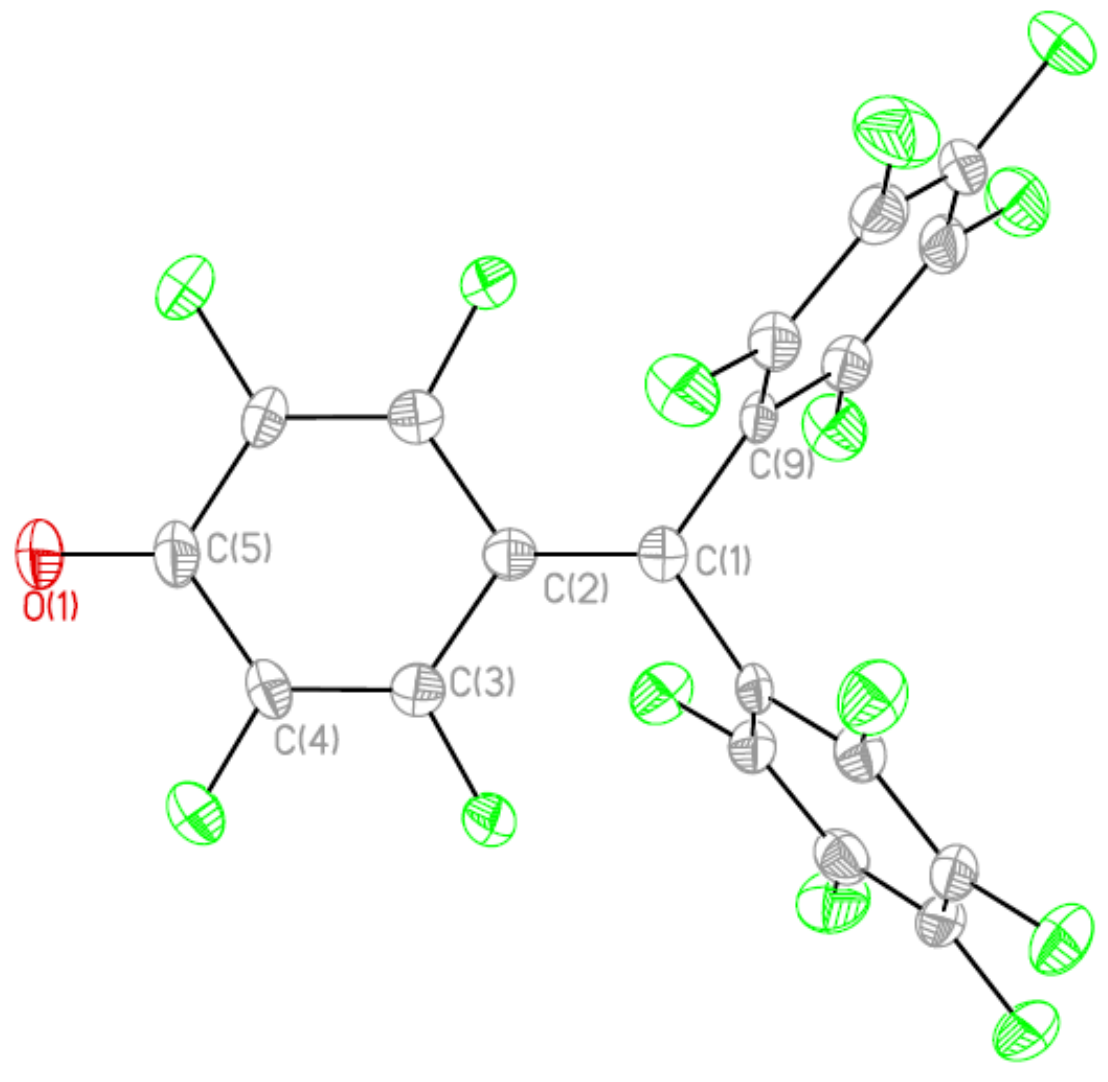

Figure 5. Solid-state structure of 6. Thermal ellipsoids are depicted at the 50\% probability level. Selected bond distances ( $\mathrm{A})$ : C(5)-O(1) 1.214(3), C(4)-C(5) 1.460(2), C(3)-C(4) 1.329(2), C(2)C(3) 1.462(2), C(1)-C(2) 1.357(3), C(1)-C(9) 1.494(2).

The addition of neat triflic acid to solid $\mathbf{1 0 H}$ at $-30{ }^{\circ} \mathrm{C}$ resulted in immediate generation of a deep maroon solution (Scheme 4). ${ }^{19}$ F NMR spectroscopy of the solution with the NMR probe set at -30 ${ }^{\circ} \mathrm{C}$ revealed three signals at $-115,-128$, and -157 ppm. The strongly downfield shift of the para and ortho fluorine atoms is consistent with the observations of Olah and co-workers for ionized $\mathbf{1}^{+}$in strongly acidic media. ${ }^{3}$ While this cation is stable for at least two hours at $-30{ }^{\circ} \mathrm{C}$, warming to room temperature results in decomposition, with ketone $\mathbf{6}$ being the main product after a sample was held overnight. 
<smiles>Oc1c(F)c(F)c(C(O)(c2c(F)c(F)c(F)c(F)c2F)c2c(F)c(F)c(F)c(F)c2F)c(F)c1F</smiles>

$10 \mathrm{H}$

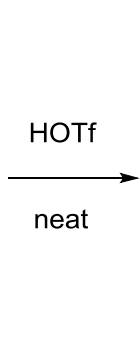<smiles>Fc1c(F)c(F)c(C(c2c(F)c(F)c(F)c(F)c2F)c2c(F)c(F)c(F)c(F)c2F)c(F)c1F</smiles>

[1][OTf]

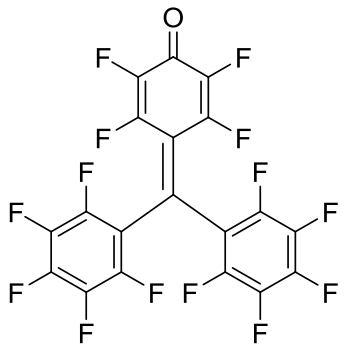

6

Scheme 4. Generation of $\mathbf{1}^{+}$as a triflate salt in neat HOTf and decomposition to 6 upon warming.

Neat triflic acid is a harsh medium and likely precludes the synthetic use of $\mathbf{1 +}$. We therefore explored its formation in organic solvents. Reacting two equivalents of $\mathrm{TfOH}$ (one to protonate the alcohol, and the second to sequester the resulting equivalent of $\mathrm{H}_{2} \mathrm{O}$ ) with $\mathbf{1 0 H}$ in orthodichlorobenzene (ODCB) resulted in the immediate formation of a dark red colored solution (Scheme 5). The ${ }^{19} \mathrm{~F}$ NMR spectrum of the reaction mixture at $-10{ }^{\circ} \mathrm{C}$ was relatively clean, with three ${ }^{19} \mathrm{~F}$ signals at $-142.5,-153.6$, and $-162.8 \mathrm{ppm}$. These signals are distinctly different from those of $\mathbf{1 0 H}$ in the same solvent and far upfield to what is consistent with a free cation, as observed with neat triflic acid. Warming to room temperature resulted in decomposition of the mixture to signals consistent with 6 as the main product after 48 hours. The data is consistent with the formation of 1OTf; with the triflate fragment remaining bound to the carbon centre.
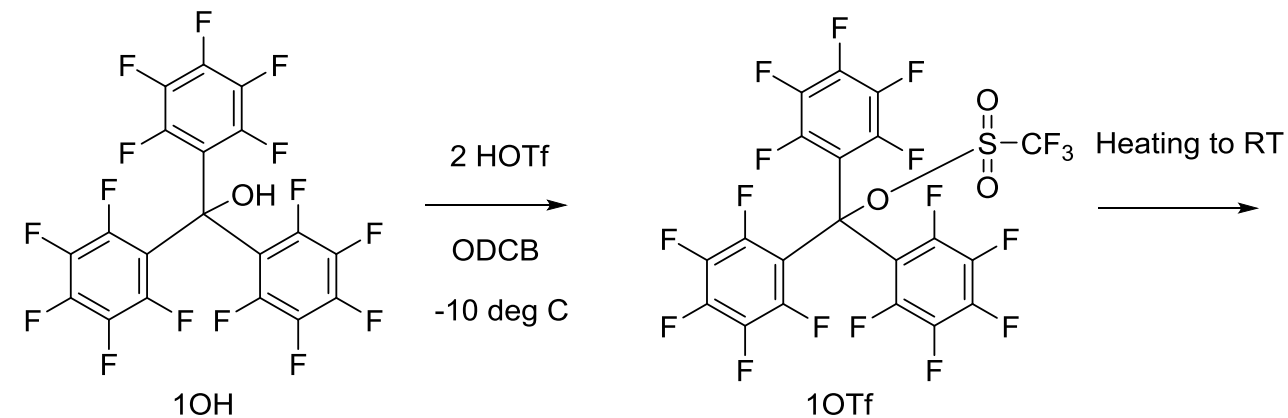<smiles>O=C1C(F)=C(F)C(=C(c2c(F)c(F)c(F)c(F)c2F)c2c(F)c(F)c(F)c(F)c2F)C(F)=C1F</smiles>

Scheme 5. Generation of 10Tf and decomposition to 6 upon warming. 
To lend support to the proposed formation of 10Tf and explore its synthetic utility, we explored its ability to act as a hydride acceptor. This is expected behavior, as triflate is an excellent leaving group bound to electron-deficient carbon.

Addition of $\mathrm{Et}_{3} \mathrm{SiH}$ as a hydride donor to a solution of 10Tf prepared from $\mathbf{1 0 H}$ and two equivalents of HOTf at $-10{ }^{\circ} \mathrm{C}$ in ortho-dichlorobenzene (Scheme 6), resulted in an NMR spectrum consistent with the presence of two species, one the regenerated alcohol $\mathbf{1 0 H}$, and the other as tris(pentafluorophenyl)methane 1H. ${ }^{16}$ Performing the reaction in toluene at low temperature allowed for simpler isolation of $\mathbf{1 H}$ in a pure form, because toluene is more easily removed under vacuum. We hypothesize that the competing reactions shown in Scheme 6 account for this distribution of products. Nevertheless, the generation of $\mathbf{1 H}$ is supportive of the generation of 1OTf. The anion is too strongly coordinating to allow ionization to $\mathbf{1}^{+}$in low dielectric media. 


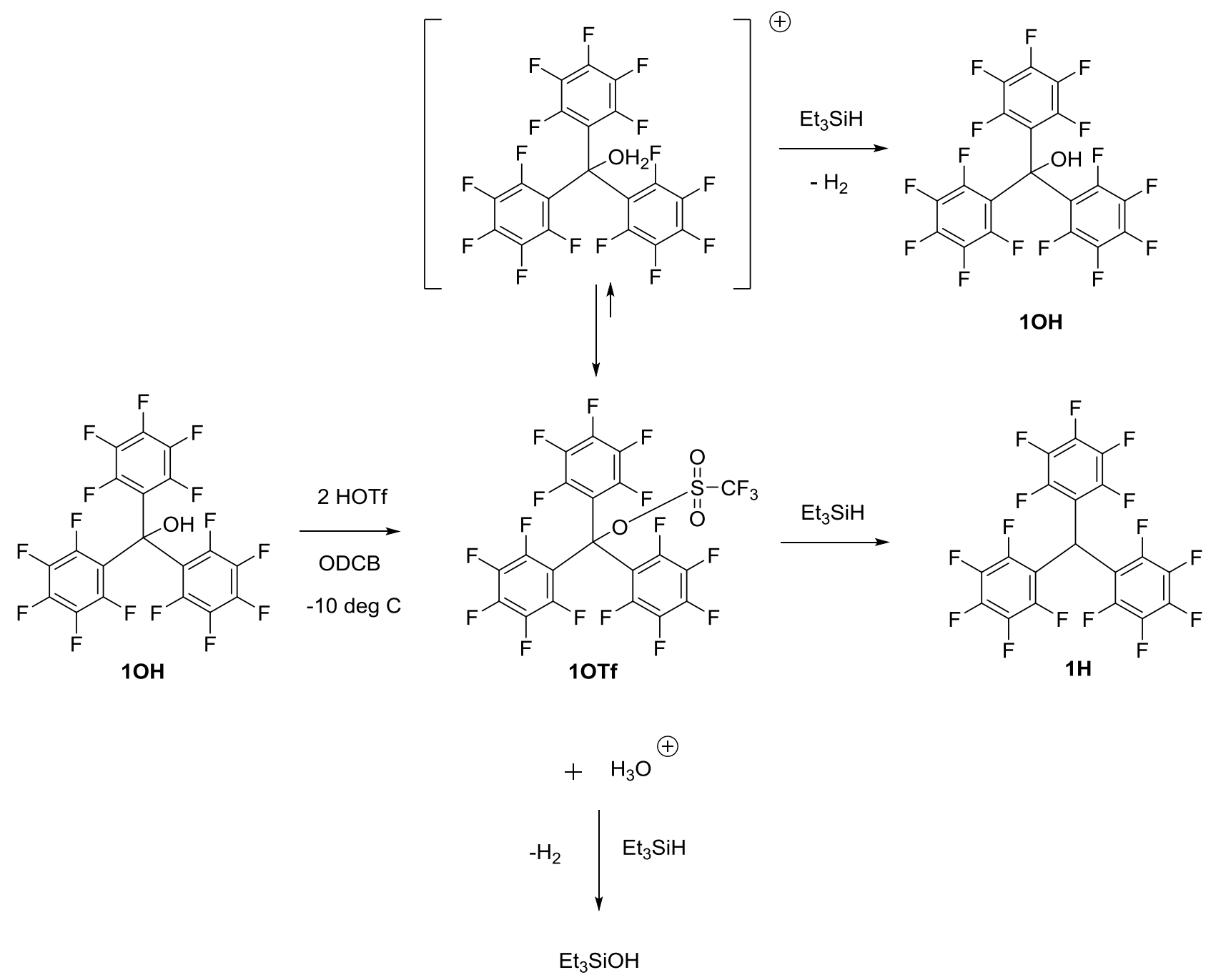

Scheme 6. Reaction pathways of 10Tf with hydride donor $\mathrm{Et}_{3} \mathrm{SiH}_{\text {. }}$

Carborane anions are much less coordinating than triflate and often allow isolation of extremely reactive cations. ${ }^{8}$ To determine if the closo-carborane anion $\left[\mathrm{HCB}_{11} \mathrm{Cl}_{11}\right]^{-}$might allow isolation of "free" $\mathbf{1}^{+}$in non-super acidic media, solid alcohol $\mathbf{1 0 H}$ was mixed with two equivalents of solid $\left[\mathrm{C}_{6} \mathrm{H}_{7}\right]\left[\mathrm{HCB}_{11} \mathrm{Cl}_{11}\right]$, the protonated benzene salt of the carborane. IR spectroscopy indicated that mixing the two solids did not result in any reaction but the addition of a drop of benzene resulted in the immediate generation of a maroon slurry, followed by immediate evaporation. IR spectroscopy of the resulting solid indicated loss of $\mathbf{1 0 H}$. The vibrations associated with stretching of $\mathrm{C}_{\text {central- }}$ $\mathrm{C}_{\mathrm{ipso}}$ bonds are shifted to lower frequencies $\left(1604-1611 \mathrm{~cm}^{-1}\right)$ compared to $\mathbf{1 O H}\left(1653 \mathrm{~cm}^{-1}\right)$. For comparison, calculated vibration frequencies using B3LYP-D3(BJ)/def2-TZVPP give 1671-1678 
$\mathrm{cm}^{-1}$ for $\mathbf{1 O H}$ and $1667 \mathrm{~cm}^{-1}$ for $\mathbf{1}^{+}$. For comparison, the IR spectrum for the trityl salt of the same carborane anion shows stretches associated with the central-ipso C-C bonds at $1577 \mathrm{~cm}^{-1}$ while the computationally predicted vibration for the trityl cation is found at $1630 \mathrm{~cm}^{-1}$. The blue shift for the perfluorinated compound is likely a result of a stronger C-C bond induced by the $\pi$ donor ability of the fluorine atoms.

Despite this evidence for the existence of $\mathbf{1}^{+}$in the solid state, it does not appear that the cation is stable in low polarity solvents. Although addition of 2 equivalents of $\left[\mathrm{C}_{6} \mathrm{H}_{7}\right]\left[\mathrm{CHB}_{11} \mathrm{Cl}_{11}\right]$ to a solution of $\mathbf{1 0 H}$ in a variety of arene solvents (toluene, benzene, ortho-dichlorobenzene) did result in the immediate formation of red solutions, they underwent decolorization within minutes and we were unable to identify any ${ }^{19} \mathrm{~F}$ NMR signals consistent with formation of $\mathbf{1}$.

\section{CONCLUSIONS}

We have demonstrated that the perfluorinated trityl cation exists in neat triflic acid. In less polar organic solvents it exists with bound triflate but nevertheless shows reactivity as a hydride acceptor with silanes. The free cation appears to exist in the solid state when the counterion is switched to an ultra-inert carborane but intrinsic decomposition prevents its full characterization. It is apparent that the para-carbon position of the $-\mathrm{C}_{6} \mathrm{~F}_{5}$ ring is the Achilles heel of this reactive species.

\section{ASSOCIATED CONTENT}


Supporting Information. Experimental details with associated ${ }^{1} \mathrm{H},{ }^{19} \mathrm{~F}$, IR spectra of synthesized compounds and reaction mixtures. Computational details and Cartesian coordinates for calculated species. X-ray crystallographic details in .cif format.

\section{AUTHOR INFORMATION}

\section{Corresponding Author}

*j.dutton@latrobe.edu.au

\section{ACKNOWLEDGMENT}

We thank The La Trobe Institute for Molecular Science for their generous funding of this project.

This work was also supported by an ARC Future Fellowship (JLD, FT16010007).

\section{REFERENCES}

(1) Olah, G. A.; Prakash, G. K.; Sommer, J. Superacids; John Wiley and Sons: New York, 1985.

(2) Filler, R.; Wang, C.; McKinney, M. A.; Miller, F. N. J. Am. Chem. Soc. 1967, 89, 1026.

(3) Olah, G. A.; Comisarow, M. B. J. Am. Chem. Soc. 1967, 89, 1027.

(4) Horn, M.; Mayr, H. Eur. J. Org. Chem. 2011, 6470.

(5) Horn, M.; Metz, C.; Mayr, H. Eur. J. Org. Chem. 2011, 6476.

(6) Lawson, J. R.; Melen, R. L. Inorg. Chem. 2017, 56, 8627.

(7) Erker, G. Dalton Trans. 2005, 1883.

(8) Reed, C. A. Acc. Chem. Res. 2010, 43, 121.

(9) Krossing, I.; Raabe, I. Angew. Chem. Int. Ed. 2004, 43, 2066.

3902.

(10) Couchman, S. A.; Wilson, D. J. D.; Dutton, J. L. Eur. J. Org. Chem. 2014, 2014,

(11) Jupp, A. R.; Johnstone, T. C.; Stephan, D. W. Dalton Trans. 2018, 47, 7029.

(12) Berlin, K. D.; Gower, L. H.; White, J. W.; Gibbs, D. E.; Strum, G. P. J. Org. Chem. 1962, 27, 3595 .

(13) Marwitz, A. J. V.; Dutton, J. L.; Mercier, L. G.; Piers, W. E. J. Am. Chem. Soc. 2011, 133, 10026.

(14) Gerasimova, T. N.; Lokshina, E. G.; Barkhash, V. A.; Vorozhtsov, N. N. Zhurnal Obschei Khimii 1967, 37, 1296.

(15) Geis, V.; Guttsche, K.; Knapp, C.; Scherer, H.; Uzun, R. Dalton Trans. 2009, 2687.

(16) Vorozhtsov, N. N.; Barkhash, V. A.; Gerasimova, T. N.; Lokshina, E. G.; Ivanova, N. G. Zhurnal Obschei Khimii 1967, 37, 1293. 